\title{
An Analysis of Crack Trapping by Residual Stresses in Brittle Solids
}

The residual stress distribution in a brittle polycrystalline solid may have a significant influence on its toughness. Grains in a state of residual compression are less likely to be fractured by a growing crack and may trap the crack front or be left behind as bridging particles (Evans et al., 1977). This paper estimates the toughness enhancement due to intergranular residual stresses, using a three-dimensional model. The residual stress is approximated as a doubly sinusoidal distribution acting perpendicular to the plane of an initially straight semi-infinite crack. An incremental perturbation method developed by Bower and Ortiz (1990) for solving three-dimensional crack problems is extended here to cracks loaded by nonuniform remote stresses. It is used to calculate the shape of the semi-infinite crack as it propagates through the doubly sinusoidal residual stress. It is shown that the local regions of compression may trap the crack front and give rise to some transient toughening. In addition, if the residual stress exceeds a critical magnitude, pinning particles may be left in the crack wake. However, for practical values of residual stress and grain size, the predicted toughness enhancement is insignificant. Furthermore, the analysis cannot account for the large bridging zones observed in experiments. It is concluded that the R-curve behavior and bridging particles observed in monolithic ceramics are caused by mechanisms other than residual stresses acting perpendicular to the crack plane.

\section{Introduction}

The toughness of a brittle material may be significantly improved by the presence of small quantities of tough particles. Two mechanisms are responsible. The particles may trap the front of a crack propagating through the matrix, causing it to bow out between them. In addition, they may be left behind in the wake of the crack, pinning its faces and carrying part of the load. Several theoretical models have been developed to predict the toughness enhancement in composite materials such as particle or fiber reinforced ceramics. The effects of crack bridging may be estimated using a simple two-dimensional model, in which the bridging zone is approximated by a distribution of pressure $p(\delta)$ acting on the crack flanks. The relationship between $p$ and the crack opening displacement $\delta$ is determined by the properties and volume fraction of the second-phase particles: for ductile particles, appropriate expressions have been given by Budiansky (1986) and Budiansky, Amazigo, and Evans (1988). The influence of frictionally constrained fibers has been modeled by Budiansky et al. (1986), Budiansky and Amazigo (1989), and Sigl and Evans (1989), among others. It is less straightforward to estimate the

Contributed by the Applied Mechanics Division of THE AMERICAN Socrety of Mechanical Engineers for publication in the ASME Journal OF APPLIED Merianics.

Discussion on this paper should be addressed to the Technical Editor, Professor Lewis T. Wheeler, Department of Mechanical Engineering, University of Honston, Houston, TX 77204-4792, and will be accepted until four months after Tinal publication of the paper itself in the ASME Journal OF APPLIED ME4hres.

Manuscript received by the ASME Applied Mechanics Division, May 22, 1991; Thal revision, Dec. 30, 1991. Associate Technical Editor: R. M. McMeeking. toughness enhancement due to crack trapping, since this requires a three-dimensional analysis. The behavior of a semiinfinite crack bypassing a single row of particles was investigated by Fares (1989), Gao and Rice (1989), and Bower and Ortiz (1990). More recently, Bower and Ortiz (1991) developed a full three-dimensional model which includes the effects of both crack trapping and bridging. These analyses predict substantial improvements in toughness: increases of 10-20 times the matrix toughness have been estimated for a brittle material reinforced by ductile particles, and 2-4 times for a fiber-reinforced solid. The predictions are in good agreement with experimental measurements by Mendelson and Fine (1974) and Krstic et al. (1981).

Crack trapping and bridging are also believed to be important toughening mechanisms in monolithic ceramics and rocks. Swanson et al. (1987), and Vekinis et al. (1990) have observed bridging particles in the wake of cracks propagating through Alumina. Vekinis and co-workers also measured a substantial increase toughness as the bridging zone developed. This toughness enhancement is difficult to estimate theoretically, since the source of the particles is not clear. It is possible to apply the models developed for composite materials, if the properties of the particles are regarded as adjustable parameters, which are determined by fitting the theory to experimental measurements. This approach was used successfully by Bower and Ortiz (1991) to predict the R-curve behavior observed in Alumina by Vekinis et al. (1990). However, it would be more satisfactory to account for the mechanisms responsible for forming the pinning particles. 
Evans et al. (1977) have suggested that bridging particles in ceramics may be attributed to the action of intergranular residual stresses. Substantial residual stresses may be set up in polycrystalline ceramics during manufacture, due to the thermal and elastic anisotropy of the grains: stresses of the order 70-150 MPa have been measured experimentally (Blendell and Coble, 1982) and stresses of a similar magnitude have been predicted theoretically (Ortiz and Molinari, 1988; Ortiz and Suresh, 1990). According to Evans and co-workers, grains in a state of residual compression are less likely to be fractured by a growing crack and may be left behind as bridging particles. They pointed out that compressive grains may be thought of as regions of increased toughness, and have proposed an expression relating the magnitude of the compressive stress and the grain size to the equivalent toughness of the particles.

This paper investigates the influence of intergranular residual stresses on the effective toughness of a brittle polycrystalline solid, using a three-dimensional model. The problem to be solved is illustrated in Fig. 1. We consider an unbounded elastic body containing a semi-infinite crack on the plane $x_{3}$ $=0$. The crack front is initially straight and parallel to the $x_{2}$-axis, at a position $x_{1}=a$. The solid is elastically homogeneous and isotropic, and is assumed to be ideally brittle with a fracture toughness $K_{c}^{\text {mat }}$. It is subjected to remote loads, which set up a uniform distribution of mode I stress intensity $K(s)$ $=K^{\infty}$ along the crack front. In addition, the solid contains a distribution of self-equilibrating residual stress. For simplicity, we consider only the component of residual stress acting perpendicular to the plane of the crack, which is approximated by

$$
\sigma_{33}\left(x_{1}, x_{2}\right)=\sigma_{r} \cos \left(2 \pi x_{1} / L_{1}\right) \cos \left(2 \pi x_{2} / L_{2}\right) .
$$

Here, $\sigma_{r}$ is the magnitude of the residual stress, and the wavelengths $L_{1}$ and $L_{2}$ are assumed to be of the order of the grain size. Thus, the $x_{3}=0$ plane is subjected to equi-distant regions of alternating tension and compression. The residual stress induces only mode I stress intensities, so the crack is not deflected from its initial plane, but once the crack starts to advance, its front does not remain straight. The compressive zones tend to trap the crack front, while the tensile regions accelerate growth. We seek to calculate the crack shape and the variation in remote stress, parameterized by $K^{\infty}$, as the crack propagates through the solid.

Some qualitative predictions may be made without analysis. Since the residual stress field is self-equilibrating, the average effective toughness is given by $K^{\infty}=K_{c}^{\text {mat }}$. In other words, if a large area of material is fractured, the strengthening effect of the compressive zones exactly cancels the weakening due to tensile regions. However, the residual stress gives rise to some transient toughening. The crack front will initially propagate rapidly through tensile regions, and will adopt a configuration such that the entire crack front is subjected to compressive loading. In order for the crack to bypass the compressive zones, it is necessary to increase the remote load so that $K^{\infty}>K_{c}^{\mathrm{mat}}$. The overall behavior of the solid is determined by the boundary conditions. If displacements are prescribed at infinity, the remote load fluctuates about the mean value $K^{\infty}=K_{c}^{\text {mat }}$. Under conditions of prescribed remote stress, the load must be increased to its maximum value so as to bypass the first set of compressive zones. Thereafter, the crack propagates rapidly through the intervening tensile regions, radiating energy in the form of elastic waves. Here, we assume remote displacement boundary conditions, and the possibility of dynamic crack growth is not considered.

A rigorous analysis of the problem in hand is not trivial. It is necessary to calculate stress intensity factors for an arbitrarily shaped crack under nonuniform loading. In addition, the shape of the crack is not known a priori, and must be determined as part of the solution. A suitable approach to solving problems of this nature has been devised by Rice $(1985,1989)$ and Gao

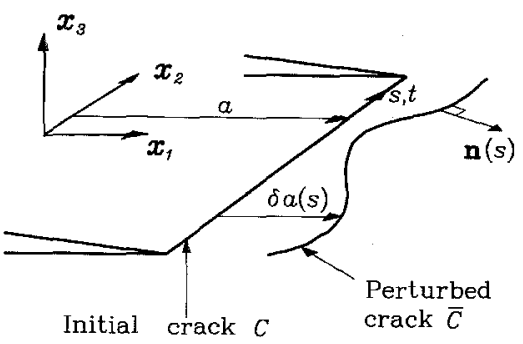

Fig. 1 Semi-infinite crack with a wavy front, showing notation and sign convention

and Rice (1986, 1987), who found a method for calculating the change in stress intensity factor caused by a small change in the geometry of a crack. Bower and Ortiz (1990) recently followed a suggestion by Rice (1989) to extend the method to arbitrarily large changes in crack shape. By applying a succes. sion of small perturbations to a crack of some convenient initial geometry and updating the stress intensity factors at each step, it is possible to find stress intensities for any arbitrarily shaped crack. This approach has two advantages over alternative numerical methods. First, the solution only involves evaluating two one-dimensional integrals around the crack front, and secondly, it is straightforward to find a change in crack shape consistent with a prescribed change in stress intensity factor.

Bower and Ortiz (1990) were only able to apply the incremental perturbation method to conditions where the crack was subjected to uniform remote stresses. It is extended here to arbitrary loading, and used to investigate the propagation of a semi-infinite crack through a doubly sinusoidal residual stress field. The analysis shows that residual stresses can give rise to some transient toughening in the solid, and may also lead to the formation of bridging particles in the crack wake, as suggested by Evans et al. (1977). However, for realistic values of residual stress and grain size, the toughening is extremely small, and no bridging particles are likely to be formed. It therefore appears that the crack bridging and R-curve behavior observed in monolithic ceramics is due to mechanisms other than crack trapping by intergranular residual stresses.

\section{Theory}

We begin by describing a method for calculating stress intensity factors on a planar crack with an arbitrarily shaped front. The procedure is based on work by Rice $(1985,1989)$ and Gao and Rice $(1986,1987)$, who devised a linear perturbation scheme to find the variation in stress intensity factor due to small changes in crack geometry. Bower and Ortiz (1990, 1991) have used this approach as the basis of a numerical method for calculating stress intensity factors for three-dimensional cracks. Their analysis considered only the special case of cracks subjected to a uniform distribution of remote stress: it is extended here to arbitrary mode I loading.

Consider an infinite solid, containing an arbitrarily shaped crack which lies in the $\left(x_{1}, x_{2}\right)$ plane, as illustrated in Fig. 1. The shape of the crack front $C$ is specified by its parametric equation $\mathbf{z}(s)$. The crack is subjected to a distribution of re mote stress, acting in the $x_{3}$ direction, which induces a mode I stress intensity factor $K(s)$ on the crack front. Assume that the stress intensity factor at a point $s$ on the crack front due to a pair of unit wedging forces acting on the crack faces at $\xi$ is given by $H(\xi, s)$, where $H$ is the crack face weight function The stress intensity factor due to the remote stress distribution can therefore be calculated by integrating the weight function over the crack faces

$$
K(s)=\int_{\Omega} H(\xi, s) p(\xi) d \xi_{1} d \xi_{2}, \quad[\xi \in \Omega] .
$$


where $p(\xi)$ is the distribution of stress in the uncracked solid, and $\Omega$ is the area bounded by the crack front. It is convenient at this point to define an additional influence function $D$ for the crack, given by

$$
D(t, s)=\lim _{\epsilon \rightarrow 0} \sqrt{\frac{\pi^{3}}{2}} \frac{H(\mathbf{z}(t)-\epsilon \mathbf{n}(t), s)}{\sqrt{\epsilon}},
$$

where $\mathbf{z}(t)$ is the position vector of a point $t$ on the crack front, and $\mathbf{n}(t)$ is a unit vector in the crack plane, pointing in a direction normal to $C$ at $t$. The influence function $D$ can be seen to be the limiting stress intensity factor as a pair of squareroot singular point loads approach the crack front: its signifieance is discussed in Bower and Ortiz (1990).

Now, imagine allowing the crack front to advance by a small increment $\delta a(s)$ n $(s)$, so that the crack occupies a new configuration $\bar{C}$, given by

$$
\overline{\mathbf{z}}(s)=\mathbf{z}(s)+\delta a(s) \mathbf{n}(s) .
$$

The stress intensity factor for the perturbed geometry becomes

$$
\bar{K}(s)=K(s)+\delta K(s),
$$

and we wish to calculate $\delta K$ at some point $s=t$ to first order in $\delta a(s)$. Following Rice $(1985,1989), \bar{C}$ is reached in two stages. First, we translate the crack by a distance $\delta a(t) \mathbf{n}(t)$ to a reference configuration $C^{\text {ref }}$, while leaving its shape unchanged. The weight function $H$ is invariant under translation, so (2) shows that the stress intensity factor on $C^{\text {ref }}$ is

$$
K^{\text {ref }}(t)=\int_{\Omega^{\text {ref }}} H(\xi, t) p(\xi-\delta a(t) \mathbf{n}(t)) d \xi_{1} d \xi_{2}, \quad\left[\xi \in \Phi^{\text {ref }}\right] .
$$

Expanding to first order in $\delta a(t)$ shows that

$$
K^{r l t}(t)=K(t)+\delta a(t) \int_{\Omega} H(\xi, t) \frac{\partial p(\xi)}{\partial \xi} \cdot \mathbf{n}(t) d \xi_{1} d \xi_{2}, \quad[\xi \in \Omega]
$$

Next, the crack is perturbed from $C^{\text {ref }}$ by an amount

$$
\delta a^{\mathrm{ref}}(s)=\delta a(s)-\mathbf{n}(s) \cdot \mathbf{n}(t) \delta a(t),
$$

so that the combined translation and perturbation reaches $\bar{C}$. Rice $(1985,1989)$ has shown that the change in stress intensity factor due to this perturbation is

$$
\delta K(t)=\frac{1}{2 \pi} \int_{C} K^{\mathrm{ref}}(s) D(s, t) \delta a^{\mathrm{ref}}(s) d s,
$$

so that, combining (7) and (9) and discarding second order terms, we find

$$
\begin{gathered}
\bar{K}(t)=K(t)+\delta a(t) \int_{\Omega} H(\xi, t) \frac{\partial p(\xi)}{\partial \xi} \cdot \mathbf{n}(t) d \xi_{1} d \xi_{2} \\
+\frac{1}{2 \pi} \int_{C} K(s) D(s, t)[\delta a(s)-\mathbf{n}(s) \cdot \mathbf{n}(t) \delta a(t)] d s+\mathcal{O}\left(\delta a^{2}\right)
\end{gathered}
$$

The process of dividing $\delta a(s)$ into a sequence of translation and perturbation appears cumbersome, but is necessary to ensure that the second integral in $(10)^{-}$be well defined. Rice (1989) has shown that $D(s, t)$ contains a singularity of order $(t-s)^{-2}$ as $s \rightarrow t$. The reference configuration $C^{\text {ref }}$ is chosen so that when the crack is perturbed from $C^{\text {ref }}$ to $\bar{C}, \delta a^{\text {ref }}(s)$ -0 as $s \rightarrow t$. The integral around the crack front in (10) may then be shown to exist as a principal value, provided that $d^{6} a^{r e t}(s) / d s$ is Hölder continuous at $s=t$.

Equation (10) extends the results given by Rice (1985) to take into account the effects of non-uniform remote loading. For the particular case of a uniform remote stress field, $\partial p(\xi)$ / $\partial \xi=0$, so the first integral vanishes, and (10) is then identical to equations given in Rice $(1985,1989)$.

Bower and Ortiz (1990) have developed a numerical method, based on (10), for solving three-dimensional crack problems. They considered only uniform remote loading, with $\partial p(\xi) / \partial \xi$ $=0$. In this case, stress intensity factors may be calculated for any arbitrarily shaped crack, by applying a succession of small perturbations to a crack with some convenient initial geometry. After each perturbation, $K(s)$ may be updated by evaluating the integral in (10), provided that $D(s, t)$ can be found. In practice, exact expressions for $D$ are known only for circular and semi-infinite cracks. However, the change in $D$ due to small changes in the shape of a crack may be calculated: Bower and Ortiz $(1990,1991)$ have shown that

$$
\begin{gathered}
\delta D(s, t)=\frac{1}{2 \pi} \int_{C} D(\lambda, t) D(s, \lambda) \\
\times\left\{\delta a(\lambda)-\frac{[\mathbf{z}(s)-\mathbf{z}(t)] \cdot[\mathbf{z}(\lambda)-\mathbf{z}(t)]}{[\mathbf{z}(t)-\mathbf{z}(s)] \cdot[\mathbf{z}(t)-\mathbf{z}(s)]} \mathbf{n}(\lambda) \cdot \mathbf{n}(s) \delta a(s)\right. \\
\left.-\frac{[\mathbf{z}(t)-\mathbf{z}(s)] \cdot[\mathbf{z}(\lambda)-\mathbf{z}(s)]}{[\mathbf{z}(t)-\mathbf{z}(s)] \cdot[\mathbf{z}(t)-\mathbf{z}(s)]} \mathbf{n}(\lambda) \cdot \mathbf{n}(t) \delta a(t)\right\} d \lambda \\
-\frac{\delta a(s)}{2 \pi} \int_{C} D(\lambda, t) D(s, \lambda) \frac{[\mathbf{z}(\lambda)-\mathbf{z}(t)] \wedge[\mathbf{z}(s)-\mathbf{z}(t)] \cdot \mathbf{k}}{[\mathbf{z}(t)-\mathbf{z}(s)] \cdot[\mathbf{z}(t)-\mathbf{z}(s)]} \\
\quad \times \mathbf{n}(\lambda) \wedge \mathbf{n}(s) \cdot \mathbf{k} d \lambda \\
-\frac{\delta a(t)}{2 \pi} \int_{C \quad} D(\lambda, t) D(s, \lambda) \frac{[\mathbf{z}(\lambda)-\mathbf{z}(s)] \wedge[\mathbf{z}(t)-\mathbf{z}(s)] \cdot \mathbf{k}}{[\mathbf{z}(s)-\mathbf{z}(t)] \cdot[\mathbf{z}(s)-\mathbf{z}(t)]} \\
\quad \times \mathbf{n}(\lambda) \wedge \mathbf{n}(t) \cdot \mathbf{k} d \lambda \\
-2 D(s, t) \frac{[\delta a(t) \mathbf{n}(t)-\delta a(s) \mathbf{n}(s)] \cdot[\mathbf{z}(t)-\mathbf{z}(s)]}{[\mathbf{z}(t)-\mathbf{z}(s)] \cdot[\mathbf{z}(t)-\mathbf{z}(s)]}+\mathcal{O}\left(\delta a^{2}\right) .
\end{gathered}
$$

Here, $\wedge$ denotes a vector product, and $\mathbf{k}$ is a unit vector in a direction perpendicular to the plane of the crack. Thus, the changes in both $K$ and $D$ due to advancing the crack by $\delta a$ may be found. By applying a succession of perturbations to a crack of some convenient initial geometry such as a circular or half-plane crack, stress intensity factors may be found for any arbitrarily shaped three-dimensional crack. For a uniform remote stress, the procedure only involves evaluating two onedimensional integrals, so this method has many advantages over alternative techniques. To date, the incremental perturbation method has only been applied to situations where the remote stress is uniform, so that $\partial p(\xi) / \partial \xi=0$. It is extended in the next section to arbitrary remote stress.

2.1 Perturbation Method for Arbitrary Remote Stress. At first sight it appears that if the remote stress is nonuniform, the main advantages of the incremental perturbation method are lost, since it becomes necessary to evaluate an integral over the crack faces during each perturbation in geometry. However, it is shown below that it is possible to reduce the first integral in (10) to a hierarchy of equations involving only the crack front, by expanding $p(\xi)$ in Taylor or Fourier series. This simple extension allows the incremental perturbation method to be used to find stress intensity factors for arbitrarily shaped cracks under any remote loading.

(a) Polynomial Expansion of Remote Stress. We begin by introducing the notation

$$
K_{\alpha}(s)=\int_{\Omega} H(s, \xi) p, \alpha(\xi) d \xi
$$

where 


$$
p,_{\alpha}=\frac{\partial p}{\partial x_{\alpha}} \quad[\alpha=1,2] .
$$

Equation (10) may therefore be rewritten as

$$
\begin{aligned}
\bar{K}(s)=K(s)+\delta a(s) n_{\alpha}(s) K_{\alpha}(s) & \\
& +\frac{1}{2 \pi} \int_{C} D(s, t) K(t) \delta a^{\mathrm{ref}}(t) d t,
\end{aligned}
$$

where $n_{\alpha}$ are the components of $\mathbf{n}$, and $\delta a^{\text {ref }}$ is given in (8). Comparison of Eqs. (12) and (2) shows that $K_{\alpha}(s)$ can be regarded, for each value of $\alpha$, as the stress intensity factor induced by a distribution of normal stress equal to $p_{, \alpha}(\mathbf{x})$. Therefore, $K_{\alpha}(s)$ can be updated in exactly the same manner as $K(s)$, i.e.,

$$
\begin{aligned}
\delta K_{\alpha}(s)=\delta a(s) n_{\beta}(s) K_{\alpha \beta}(s) & \\
& +\frac{1}{2 \pi} \int_{C} D(s, t) K_{\alpha}(t) \delta a^{\mathrm{ref}}(t) d t,
\end{aligned}
$$

where

$$
K_{\alpha \beta}(s)=\int_{\Omega} H(s, \xi) p,,_{\alpha \beta}(\xi) d \xi .
$$

Proceeding inductively, it follows that a hierachy of stress intensity like functions may be defined by

$$
K_{\alpha_{1} \ldots \alpha_{k}}(s)=\int_{\Omega} H(s, \xi) p, \alpha_{1} \ldots \alpha_{k}(\xi) d \xi_{1} d \xi_{2}, \quad\left(\alpha_{k}=1,2\right),
$$

and each function may be updated using the recurrence relation

$$
\begin{aligned}
\delta K_{\alpha_{1} \ldots \alpha_{k}}(s)=\delta a(s) & n_{\alpha_{k+1}}(s) K_{\alpha_{1} \ldots \alpha_{k} \alpha_{k+1}}(s) \\
& +\frac{1}{2 \pi} \int_{C} D(s, t) K_{\alpha_{1} \ldots \alpha_{k}}(t) \delta a^{\text {ref }}(t) d t .
\end{aligned}
$$

If $p(\mathbf{x})$ is a polynomial of order $m$, then

$$
K_{\alpha_{1} \ldots \alpha_{m+1}}(s)=0 \text {, }
$$

and the hierarchy can be truncated at the $m$ th term. Special cases are those of a constant pressure, which requires only one term in the hierarchy; of a linear pressure, which requires two terms; of a quadratic pressure, which requires three terms, and so on.

Equations (17), (18), and (11) allow the incremental perturbation method described in the previous section to be applied to nonuniform remote loading. The solution begins with a crack of some convenient reference geometry, for which the crack face weight function $H(\xi, s)$ is known. Two suitable examples are a circular crack, or a half-plane crack. The remote stress is approximated by a polynomial of degree $n$, and Eq. (17) is used to set up each $K_{\alpha_{1} \ldots \alpha_{n+1}}(s)$. Stress intensity factors may then be found for any other crack in the same plane by advancing the crack front in a succession of small steps until the required geometry is reached. At each increment, the hierarchy of $K_{\alpha_{1} \ldots \alpha_{n+1}}$ is updated using (18), and the influence function $D$ is updated using (11). This procedure only involves evaluating one-dimensional integrals around the crack front.

(b) Fourier Expansion of Remote Stress. Equation (10) may also be reduced to one-dimensional integrals by taking Fourier transforms of the remote stress. To this end, let $\hat{K}(s$, $\lambda$ ) be the distribution of stress intensity factors due to stresses of the form $p(\xi)=\exp (i \lambda \cdot \xi)$, where $\lambda$ is the wave number vector and $i=\sqrt{-1}$. Explicitly,

$$
\hat{K}(s, \lambda)=\int_{\Omega} H(s, \xi) \exp (i \lambda \cdot \xi) d \xi_{1} d \xi_{2} .
$$

Using the notation introduced in the preceding section, define

$$
\hat{K}_{\alpha}(s, \lambda)=i \lambda_{\alpha} \int_{\Omega} H(s, \xi) \exp (i \lambda \cdot \xi) d \xi_{1} d \xi_{2}=i \lambda_{\alpha} \hat{K}(s, \lambda) .
$$

Therefore, from the general result (10) we obtain the following remarkably simple expression:

$\delta \hat{K}(s, \lambda)=\delta a(s) i \lambda_{\alpha} n_{\alpha}(s) \hat{K}(s, \lambda)$

$$
+\frac{1}{2 \pi} \int_{C} D(s, t) \hat{K}(t, \lambda) \delta a^{\mathrm{ref}}(t) d t .
$$

Consider now an arbitrary distribution of stress defined by its Fourier representation

$$
p(\mathbf{x})=\iint \hat{p}(\lambda) \exp (i \lambda \cdot \mathbf{x}) d \lambda_{1} d \lambda_{2} .
$$

By superposition, it follows that

$$
K(s)=\iint \hat{p}(\lambda) \hat{K}(s, \lambda) d \lambda_{1} d \lambda_{2},
$$

so that the change in stress intensity factor on the crack fromt due to the perturbation in geometry is found to be

$$
\delta K(s)=\iint \hat{p}(\lambda) \delta \hat{K}(s, \lambda) d \lambda_{1} d \lambda_{2},
$$

where $\delta \hat{K}(s, \lambda)$ is given by (22).

Thus, each Fourier component may be updated following a perturbation in crack geometry by evaluating an integral around the crack front. Remarkably, each Fourier component may be updated independently of the rest. The Fourier expansion method is most useful when $\hat{p}(\lambda)$ can be approximated by a finite number of components. If, in addition $p(x)$ is periodic, then fast Fourier transform techniques can be used.

(c) Semi-Infinite Crack in a Doubly Sinusoidal Stress Field. In this paper, we investigate the propagation of a semiinfinite crack through a doubly sinusoidal residual stress field. In principle, it would be possible to approximate the residual stress using a Taylor or Fourier series, and update the stress intensities using the procedure described in the preceding sections. However, it proves more convenient to derive directly expressions analogous to (18) and (22) for a doubly sinusoidal stress.

We therefore consider a semi-infinite crack, which occupies the region $\left(x_{1}<a, x_{3}=0\right)$, Fig. 1 , and is loaded by the residual stress field given in (1). For the straight crack, the residual stress induces a stress intensity factor

$$
\begin{array}{r}
K^{\mathrm{res}}(s)=\int_{-\infty}^{a} \int_{-\infty}^{+\infty} H\left(a-x_{1}, s-x_{2}\right) \sigma_{r} \cos \left(2 \pi x_{1} / L_{1}\right) \\
\times \cos \left(2 \pi x_{2} / L_{2}\right) d x_{2} d x_{1} .
\end{array}
$$

Here,

$$
H\left(\xi_{1}, \xi_{2}\right)=\sqrt{\frac{2 \xi_{1}}{\pi^{3}}} \frac{1}{\xi_{1}^{2}+\xi_{2}^{2}}
$$

is the crack face weight function for a half-plane crack. The change in $K^{\text {res }}(s)$ due to advancing the crack by $\delta a$ is given by (10). The result may be simplified by defining the following stress intensity factor like functions on the crack front:

$$
\begin{gathered}
K_{1}(s)=-\frac{2 \pi}{L_{1}} \int_{-\infty}^{a} \int_{-\infty}^{+\infty} H\left(a-x_{1}, s-x_{2}\right) \sigma_{r} \\
K_{2}(s)=-\frac{2 \pi}{L_{2}} \int_{-\infty}^{a} \int_{-\infty}^{+\infty} H\left(a-x_{1}, s-x_{2}\right) \sigma_{r} \\
K_{12}(s)=\frac{4 \pi^{2}}{L_{1} L_{2}} \int_{-\infty}^{a} \int_{-\infty}^{+\infty} H\left(a-x_{1}, s-L_{1}\right) \cos \left(2 \pi x_{2} / L_{2}\right) d x_{2} d x_{1} \\
\times \sin \left(2 \pi x_{1} / L_{1}\right) \sin \left(2 \pi x_{2} / L_{2}\right) d x_{2} d x_{1}
\end{gathered}
$$

so that the expression for $\delta K^{\text {res }}(s)$ may be written 
$\delta K^{\mathrm{res}}(s)=\left(n_{1}(s) K_{1}(s)+n_{2}(s) K_{2}(s)\right) \delta a(s)$

$$
+\frac{1}{2 \pi} \int_{-\infty}^{+\infty} K^{\mathrm{res}}(t) D(t, s) \delta a^{\mathrm{ref}}(t) d t .
$$

Since the residual stress field is periodic with wavelength $L_{2}$ in the $x_{2}$ direction, it may be assumed that the crack front also remains periodic. Consequently, the integral in (31) may be reduced to one wavelength of the crack front, by writing

$\delta K^{\text {res }}(s)=\left(n_{1}(s) K_{1}(s)+n_{2}(s) K_{2}(s)\right) \delta a(s)$

$$
+\frac{1}{2 \pi} \int_{0}^{c} K^{\mathrm{res}}(t) \mathrm{D}(t, s) \delta a^{\mathrm{ref}}(t) d t,
$$

where $c$ is the arc length of one period of the crack front, and

$$
\mathbf{D}(s, t)=\sum_{m=-\infty}^{\infty} D(s+m c, t) .
$$

In addition, each $K_{\alpha \beta}$ may be updated as follows:

$$
\begin{aligned}
\delta K_{1}(s)=\left(-n_{1}(s) \lambda_{2}^{2} K^{\mathrm{res}}(s)+n_{2}(s) K_{\mathrm{1} 2}(s)\right) \delta a(s) & \\
& +\frac{1}{2 \pi} \int_{0}^{c} K_{1}(t) \mathbf{D}(t, s) \delta a^{\mathrm{ref}}(t) d t
\end{aligned}
$$

$$
\begin{aligned}
\delta K_{2}(s)=\left(n_{1}(s) K_{12}(s)-\right. & \left.n_{2}(s) \lambda_{1}^{2} K^{\mathrm{res}}(s)\right) \delta a(s) \\
& +\frac{1}{2 \pi} \int_{0}^{c} K_{2}(t) \mathbf{D}(t, s) \delta a^{\mathrm{ref}}(t) d t
\end{aligned}
$$

$\delta K_{12}(s)=-\left(n_{1}(s) \lambda_{1}^{2} K_{2}(s)+n_{2}(s) \lambda_{2}^{2} K_{1}(s)\right) \delta a(s)$

$$
+\frac{1}{2 \pi} \int_{0}^{c} K_{12}(t) \mathbf{D}(t, s) \delta a^{\mathrm{ref}}(t) d t,
$$

where $\lambda_{1}=2 \pi / L_{1}$ and $\lambda_{2}=2 \pi / L_{2}$. The influence functions $D(t, s)$ and $\mathbf{D}(s, t)$ for the straight crack may be found using (3) and (27), and are given by

$$
\begin{gathered}
D(t, s)=\frac{1}{(t-s)^{2}} \\
\mathbf{D}(t, s)=\frac{1}{(c / \pi)^{2} \sin ^{2} \pi(t-s) / c} .
\end{gathered}
$$

The results enable stress intensity factors to be found for a planar semi-infinite crack with an arbitrarily shaped periodic front. Each $K_{\alpha \beta}$ may be evaluated in closed form for the straight crack, and are found to be

$$
\begin{gathered}
K^{\mathrm{res}}(s)=A \cos \left(2 \pi a / L_{1}-\theta\right) \cos \left(2 \pi s / L_{2}\right), \\
K_{1}(s)=-\frac{2 \pi}{L_{1}} A \sin \left(2 \pi a / L_{1}-\theta\right) \cos \left(2 \pi s / L_{2}\right), \\
K_{2}(s)=-\frac{2 \pi}{L_{2}} A \cos \left(2 \pi a / L_{1}-\theta\right) \sin \left(2 \pi s / L_{2}\right), \\
K_{12}(s)=\frac{4 \pi^{2}}{L_{1} L_{2}} A \sin \left(2 \pi a / L_{1}-\theta\right) \sin \left(2 \pi s / L_{2}\right), \\
A=\frac{\sigma_{r}}{\sqrt{\pi}} \frac{\sqrt{L_{1} L_{2}}}{\left(L_{1}^{2}+L_{2}^{2}\right)^{1 / 4}}, \quad \theta=\frac{1}{2} \tan ^{-1}\left(L_{2} / L_{1}\right) .
\end{gathered}
$$

2.2 Application to Crack Trapping by Residual Stresses. Finally, we may apply the procedure developed in the preceding sections to investigate crack growth through a doubly sinusoidal stress field. We now assume that the halfplane crack shown in Fig. 1 is subjected to a combination of a uniform remote stress, together with the residual stress distribution given in (1). The crack front is initially straight, and is at a position $a=5 L_{1} / 16$. The remote load therefore induces a uniform stress intensity factor $K^{\text {load }}(s)=K^{\infty}$, while Eq. (37) shows that initially $K^{\text {res }}(s)=0$. When $K^{\infty}$ reaches the toughness of the material $K_{c}^{\mathrm{mat}}$, the crack starts to propagate. Thereafter, the shape of the crack is determined from the condition that $K(s) \leq K_{c}^{\text {mat }}$ over its entire front. The crack shape is calculated here by applying a succession of small perturbations to the crack front, and calculating the crack advance consistent with the fracture criterion at each step. This condition leads to an integral equation for $\delta a(s)$

$$
\begin{aligned}
& 0= \delta K^{\mathrm{load}}(s)+\delta K^{\mathrm{res}}(s) \\
&=\frac{\delta K^{\infty}}{K^{\infty}} K^{\mathrm{load}}(s)+\left(n_{1}(s) K_{1}(s)+n_{2}(s) K_{2}(s)\right) \delta a(s) \\
&+\frac{1}{2 \pi} \int_{0}^{c}\left(K^{\text {load }}(t)+K^{\mathrm{res}}(t)\right) \mathrm{D}(t, s) \\
& \quad \times(\delta a(t)-\mathbf{n}(s) \cdot \mathrm{n}(t) \delta a(s)) d t .
\end{aligned}
$$

Here, the first term represents the change in $K(s)$ due to an increment in the remote load, and the remaining terms give $\delta K$ due to changing the crack shape. The values of $K_{1}$ and $K_{2}$ for the straight crack are given in Eqs. (39) to (42), while D is given in (38). In principle, it would be possible to specify an increment in remote load $\delta K^{\infty}$, and then use (45) to calculate the corresponding change in crack shape. However, this causes numerical difficulties when the crack is propagating under decreasing remote load. It is preferable to leave $\delta K^{\infty}$ as an unknown, and enforce an additional constraint on the maximum crack advance. Here, we have set $\delta a_{\max }=L_{1} / 500$.

The solution of (45) is complicated by the possibility that parts of the crack front may be trapped by compressive residual stress, and may arrest. Over arrested zones, it is necessary to enforce the "no healing" constraint, $\delta a(s)=0$. The positions of the arrested regions are not known a priori, and must be determined by iteration. Here, we use the following procedure. Each increment is begun with a suitable guess for the new arrested regions, and (45) is used to determine the corresponding crack advance. The solution is then checked to ensure that $\delta a(s) \geq 0$ over the entire crack front: if a negative crack advance is found at any point, that part of the crack is subsequently assumed to arrest. In addition, the condition that $K(s) \leq K_{c}^{\text {mat }}$ is checked over arrested portions: if this requirement is violated, the appropriate point is allowed to advance. A solution is found to (45) with the new approximation to the crack configuration, and the iteration is continued until successive approximations for the arrested zones agree.

Once a suitable solution has been found, $K^{\text {load }}$ is updated using

$$
\begin{aligned}
\delta K^{\text {load }}(s)=\frac{\partial K^{\infty}}{K^{\infty}} K^{\text {load }}(s) & +\frac{1}{2 \pi} \int_{0}^{c} K^{\text {load }}(t) \mathbf{D}(t, s) \\
& \times(\delta a(t)-\mathbf{n}(s) \cdot \mathbf{n}(t) \delta a(s)) d t,
\end{aligned}
$$

while the change in $K^{\text {res }}$ and each $K_{\alpha \beta}$ are calculated from (39) to (41). Finally, $D$ is updated using (11). This process is repeated to progressively march the crack front through the residual stress field.

A suitable numerical method for solving the integral Eq. (45) is described in detail by Bower and Ortiz (1990), and will only be briefly summarized here. One wavelength of the crack front is divided into $m$ elements, with three nodes $s_{2 k-2}, s_{2 k-1}$ and $s_{2 k}$ on the $k$ th element. In addition, $2 m-1$ collocation points are defined, such that $s_{j}<t_{j}<s_{j-1}$. The geometry of the crack front is specified in terms of the coordinates of each node and collocation point, and the crack advance is approximated using Hermitian interpolation between values of $\delta a\left(S_{2 k}\right)$ and $d \delta a\left(s_{2 k}\right) / d s$ for $k=1,2 \ldots m$. The variation of $K^{\text {load; }}$ $K^{\text {res }}$ and each $K_{\alpha \beta}$ is approximated by means of a Lagrangian interpolation between nodal values. Similarly, to approximate the weight function $D$, we write 


$$
\begin{gathered}
\mathbf{D}(s, t)=\frac{\mathbf{d}(s, t)(\sinh 2 \pi \gamma) /(2 \pi \gamma)}{\left(L_{2} / \pi\right)^{2}\left(\sin ^{2} \pi \eta+\sinh ^{2} \pi \gamma\right)}, \\
\gamma=\left(z_{1}(s)-z_{1}(t)\right) / L_{2} ; \quad \eta=\left(z_{2}(s)-z_{2}(t)\right) / L_{2},
\end{gathered}
$$

and use three point Lagrangian interpolation between nodal values to approximate the function $d$. The integrand in (46) may then be expressed in terms of nodal values of $\delta a$ by means of the adopted interpolation functions. As a result, the integrals may be evaluated explicitly, and the integral equation may be written in the linear form:

$$
0=\frac{\delta K^{\infty}}{K^{\infty}} K\left(t_{j}\right)+\sum_{i=0}^{m} W_{i}\left(t_{j}\right) \delta a\left(s_{2 i}\right)+V_{i}\left(t_{j}\right) \delta a^{\prime}\left(s_{2 i}\right),
$$

where $W_{i}$ and $V_{i}$ are integration weights depending on the current crack configuration. These functions are given in Bower and Ortiz (1990). Equation (48) may then be solved for the nodal values of $\delta a$ and $\delta a^{\prime}$. Subsequently, the numerical approximation to $\mathbf{D}$ must be updated: an appropriate procedure is described in Bower and Ortiz (1990). A 24 element mesh was used in all the numerical simulations reported below.

\section{Results and Discussion}

The toughening effect of the residual stress distribution can be expressed in terms of two dimensionless groups

$$
\frac{K_{c}^{\mathrm{eff}}}{K_{c}^{\mathrm{mat}}}=F\left(\frac{\sigma_{r} \sqrt{L_{1} L_{2}}}{K_{c}^{\mathrm{mat}}\left(L_{1}^{2}+L_{2}^{2}\right)^{1 / 4}}, \frac{L_{1}}{L_{2}}\right) .
$$

For the particular case $L_{1} / L_{2}=0$, the toughening may be calculated exactly. The residual stress then varies sinusoidally in a direction perpendicular to the crack front, but is uniform in the $x_{2}$-direction. The crack front remains straight, so that the remote load induces a uniform stress intensity of magnitude $K^{\text {load }}=K^{\infty}$, while $K$ due to the residual stress is

$$
K^{\mathrm{res}}(s)=\frac{\sigma_{r} \sqrt{L_{1}}}{\sqrt{\pi}} \cos \left(2 \pi a / L_{1}-\pi / 4\right) .
$$

Applying the fracture criterion $K^{\text {load }}+K^{\text {res }}=K_{c}^{\text {mat }}$ gives the variation of the remote load as a function of the position of the crack front $a$

$$
\frac{K^{\infty}}{K_{c}^{\mathrm{mat}}}=1-\frac{\sigma_{r} \sqrt{L_{1}}}{\sqrt{\pi} K_{c}^{\mathrm{mat}}} \cos \left(2 \pi a / L_{1}-\pi / 4\right) .
$$

This result confirms the qualitative prediction of the influence of residual stress made in the introduction. The average energy release rate is unchanged but the load fluctuates about the mean. In order for unstable fracture to occur, it is necessary to increase $K^{\infty}$ to its maximum value. If the effective toughness of the solid is taken as the maximum value of $K^{\infty}$, then we find that

$$
K_{c}^{\text {eff }}=K_{c}^{\text {mat }}+\sigma_{r} \sqrt{L_{1} / \pi} .
$$

Evans et al. (1977) used a similar model to estimate the toughness enhancement, but assumed a square wave variation of residual stress instead of the sinusoid used here. Their analysis also gives $K_{c}^{\text {eff }}-K_{c}^{\text {mat }}=\alpha \sigma_{r} \sqrt{L_{1}}$, but the value of $\alpha$ they predict is twice that in (52).

For values of $L_{1} / L_{2}>0$, the toughening must be calculated numerically. In practice, the wavelengths $L_{1}$ and $L_{2}$ are both likely to be of the order of the grain size of the material, so detailed computations will only be presented here for $L_{1}=L_{2}$ $=L$. Predicted crack shapes are shown in Fig. 2 for three values of $\sigma_{r} \sqrt{L} / K_{c}^{\text {mat }}$. Only one wavelength of the crack front has been plotted in each case, since the crack front has been constrained to remain periodic with wavelength $L$ in the $x_{2}$ direction. The sequence of crack shapes also repeats every half wavelength in the $x_{1}$-direction. The positions of maximum tensile and compressive stress are also marked on Fig. 2 for reference. The behavior of the crack during propagation is governed by the magnitude of the residual stress and grain size. For values of $\sigma_{r} \sqrt{L} / K_{c}^{\text {mat }}$ less than 0.85 , the crack front remains almost straight, and no crack arrest is observed. As expected, regions of the crack which propagate through tensile zones tend to bow out ahead of regions in compression. For $\sigma_{r} \sqrt{L} / K_{\mathrm{c}}^{\text {mat }}>0.85$, the residual stresses are sufficient to arrest parts of the crack front, as shown in Fig. $2(b)$. If $\sigma_{r} \sqrt{L} / K_{c}^{\text {nat }}$

\section{Direction of propagation}
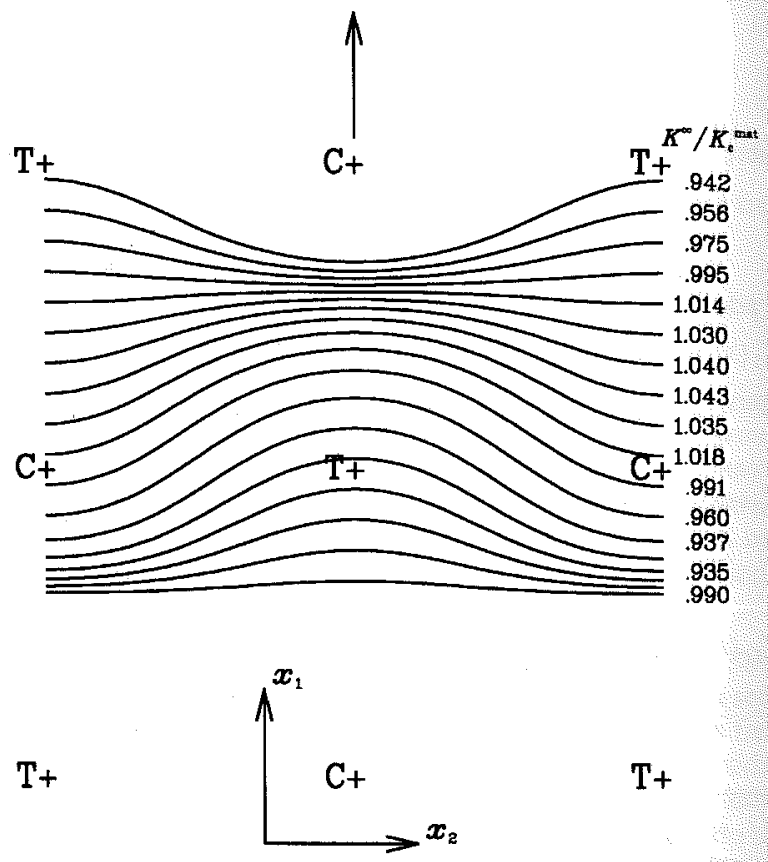

Fig. 2(a) Successive crack front profiles for a crack propagating through doubly sinusoidal stress. Residual stress amplitude $\sigma_{r} \sqrt{L} / K_{c}^{\text {mat }}=0.71$ Points marked $T$ and $C$ correspond to locations of maximum tension and compression.
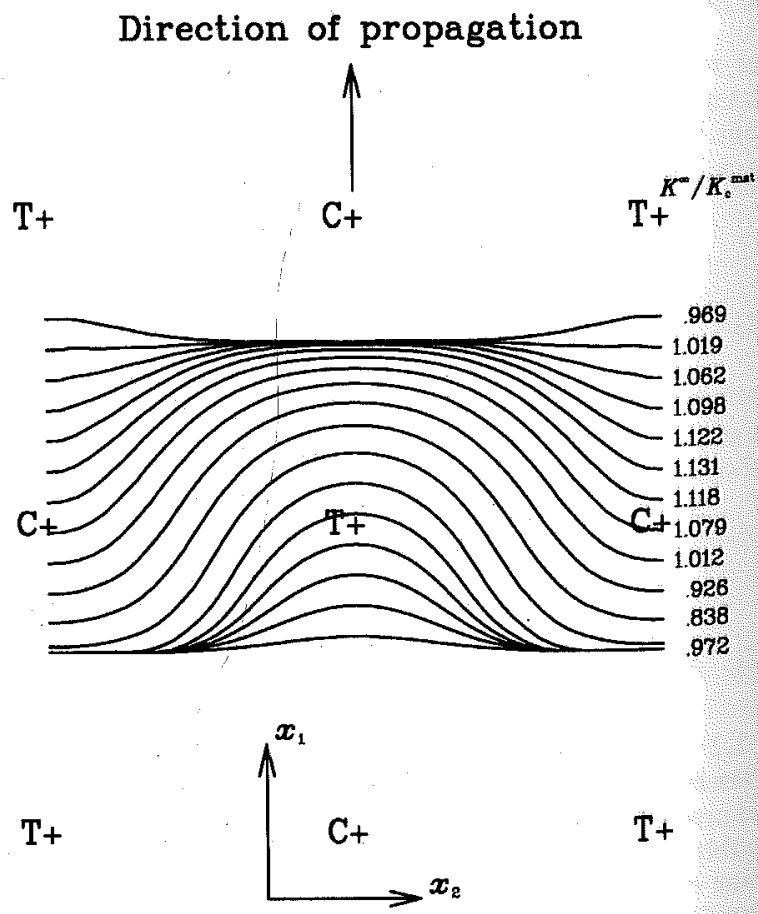

Fig. 2(b) Successive crack front profiles for a crack propagating through doubly sinusoidal stress. Residual stress amplitude $\sigma_{r} \sqrt{L} / K_{c}^{\text {mat }}=1.41$ Points marked $T$ and $C$ correspond to locations of maximum tension and compression. 
Direction of propagation

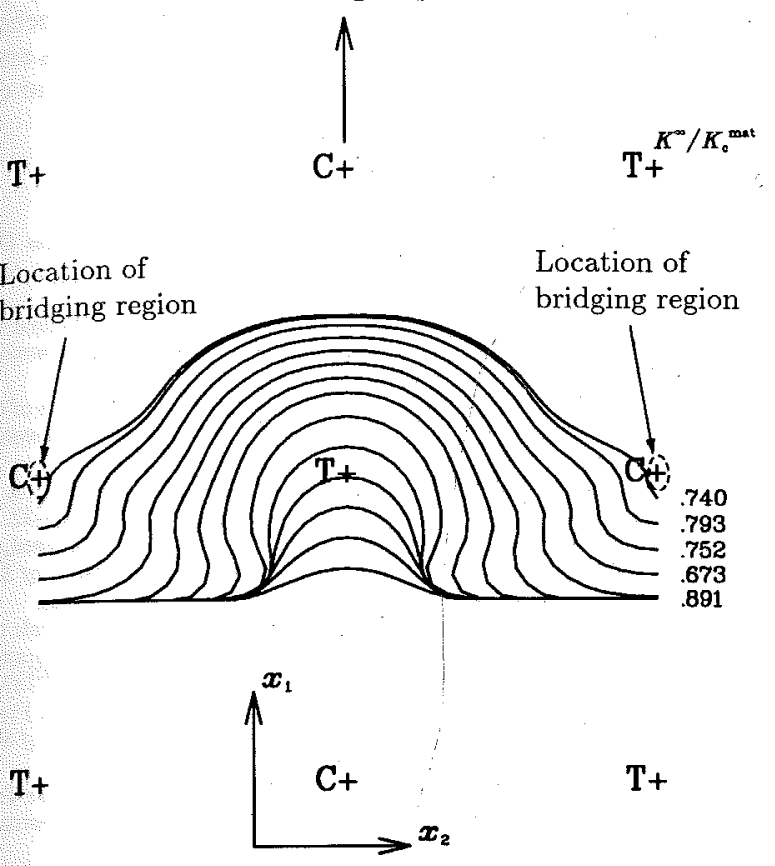

Fig. 2(c) Successive crack Iront profiles for a crack propagating through doubly sinusoidal stress. Residual stress amplitude $\sigma_{r} \sqrt{L} / K_{c}^{\text {mat }}=2.3$. Points marked $\mathrm{T}$ and $\mathrm{C}$ correspond to locations of maximum tension and compression.

exceeds 2.1 , then the compressive stress completely pins small regions of the crack, so that parts of the crack front on the far side of the pinned region join up leaving a small bridging particle in the crack wake. This behavior is illustrated in Fig. $2(c)$. The result confirms that residual stresses acting perpendicular to the crack plane may be responsible for the formation of pinning particles, as suggested by Evans et al. (1977). There are some numerical difficulties associated with applying the perturbation method to a situation where two points on a crack front coalesce (Bower and Ortiz, 1991), so Fig. 2(c) only shows the sequence of crack fronts up to the formation of the first row of pinning particles. This is not a serious restriction, for it is shown below that for practical values of $\sigma_{r}$ and $L$, bridging particles are unlikely to be formed.

It should be recognized that while the crack is propagating into tensile zones under decreasing remote stress, its shape is not necessarily unique. A detailed study of the stability and uniqueness of the crack shape has not been attempted, but it is possible to make some qualitative predictions. Rice (1985) has discussed the stability of a straight, semi-infinite crack propagating into a region of increasing stress. In his analysis, he assumes that the stress is uniform in the $x_{2}$-direction but increases in a direction perpendicular to the crack front. If the erack remains straight, the stress intensity factor increases at a constant rate $\partial K^{\infty} / \partial a=\eta$. Rice showed that if the crack is perturbed slightly from its straight configuration, there exists a critical wavelength $c_{\text {crit }}=\pi K^{\circ} / \eta$ above which perturbations tend to grow. This criterion may be used to estimate conditions for stability of a crack subjected to a doubly sinusoidal stress of wavelength $L$. The stress intensity induced by the residual stress is given in Eq. (37), and its gradient $\partial K^{\mathrm{res}} / \partial a=K_{1}(s)$ is given in (39). The critical wavelength of unstable perturbations is thus of the order

$$
c_{\text {crit }} \approx \frac{K_{c}^{\mathrm{mat}} \sqrt{L} \sqrt{\pi}}{2^{3 / 4} \sigma_{r}}
$$

Here, the crack is constrained to remain periodic, so the wave-
$K^{\infty} / K_{\mathrm{c}}^{\mathrm{mat}}$

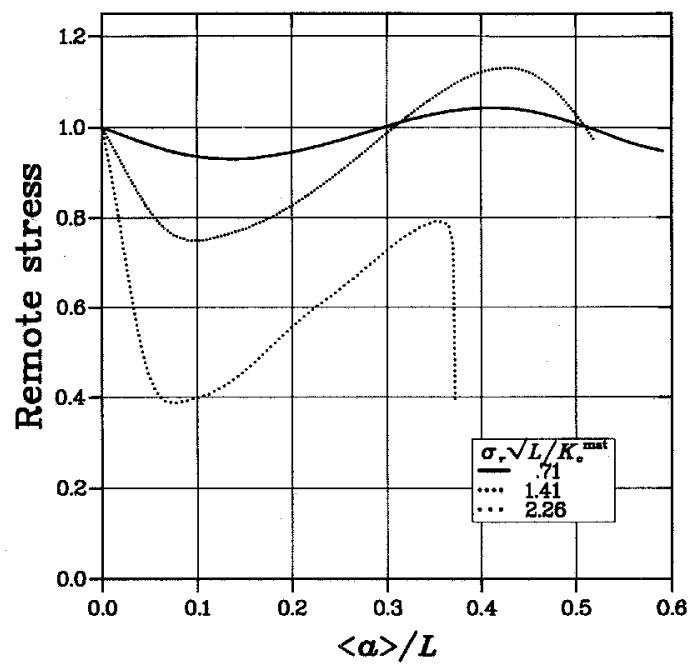

Crack advance

Fig. 3 Variation of remote stress with average crack advance for the cracks shown in Fig. 2

length of any unstable perturbations cannot exceed $L$. Putting $c_{\text {crit }}=L$ shows that instabilities may form if

$$
\frac{\sigma_{r} \sqrt{L}}{K_{c}^{\text {mat }}}>\frac{\sqrt{\pi}}{2^{3 / 4}}
$$

In the numerical simulations, the onset of crack arrest is observed to occur at a similar magnitude of residual stress.

The variation of remote stress intensity factor is plotted in Fig. 3, for the three values of $\sigma_{r} \sqrt{L} / K_{c}^{\text {mat }}$ used in the simulations shown in Fig. 2. The load is shown as a function of $\langle a\rangle / L$, where $\langle a\rangle$ is the average crack advanced, calculated using the area swept by one wavelength of the crack front. For $\sigma_{r} \sqrt{L} /$ $K_{c}^{\text {mat }}=2.3$, results are shown only up to the formation of the first row of pinning particles: the remote stress drops sharply as the crack joins up on itself, and never exceeds $K_{c}^{\text {mat }}$. If the analysis were continued beyond this point, the constraining effect of the pinning particles in the crack wake would increase the remote stress above $K_{c}^{\text {mat }}$. In the remaining cases, the load fluctuates about a mean value, such that the average energy release rate is equal to the toughness of the matrix. The maximum value of $K^{\infty}$ may be interpreted as the effective toughness of the brittle solid, since it would be necessary to increase the remote stress beyond the maximum value to produce unstable fracture.

The effective toughness has been plotted as a function of the dimensionless residual stress amplitude in Fig. 4. The result for $L_{1} / L_{2}=0$, given in Eq. (4), is also shown for comparison. Only a small increase in toughness is caused by the residual stress distribution considered here. Using practical values of $\sigma_{r}=150 \mathrm{NMm}^{-2} ; L=10 \mu \mathrm{m} ; K_{c}^{\mathrm{mat}}=5 \mathrm{MNm}^{-3 / 2}$ gives $\sigma_{r} \sqrt{L} / K_{c}^{\text {mat }}=0.095$ : the corresponding toughness enhancement is $K_{c}^{\text {eff }} / K_{c}^{\text {mat }}=1.005$. Furthermore, the analysis predicts that no pinning particles are formed under these conditions. However, in practice bridging particles have been observed up to 1000 grain diameters behind the crack front, and a toughness increase of up to 30 percent has been measured as the bridging zone is established (Vekinis et al., 1990). It therefore appears that the crack bridging and R-curve behavior observed in monolithic ceramics must be attributed to mechanisms other than residual stresses acting perpendicular to the crack plane.

The results presented here should not be taken as an indication that intergranular residual stresses have no influence on the fracture of brittle materials. Our analysis has considered only stresses acting perpendicular to the crack plane: residual 


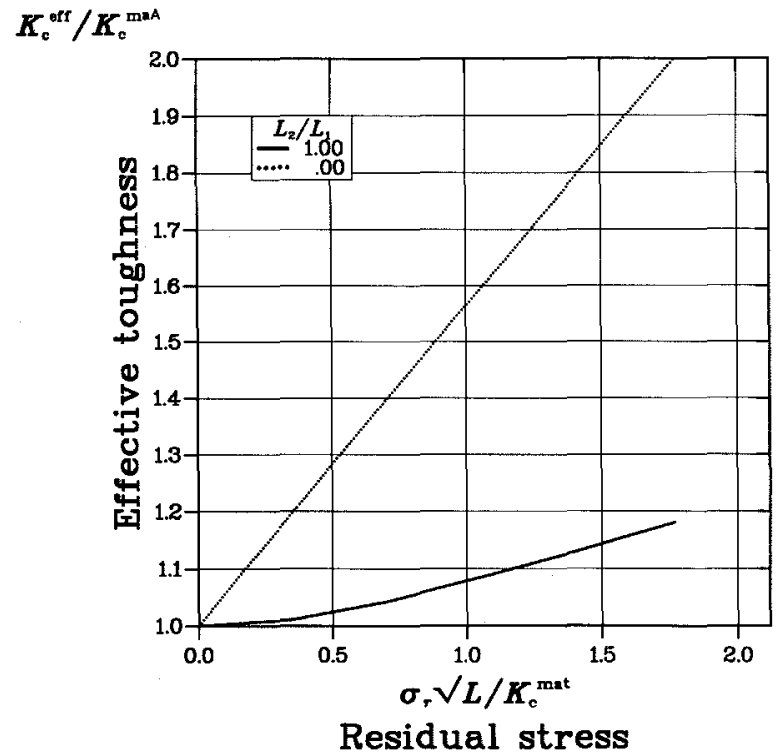

Fig. 4 Variation of effective toughness with residual stress amplitude

shear stresses may also contribute to the formation of bridging particles and cause some toughening, by deflecting the crack around grains. In addition, toughening is known to be caused by the energy dissipated by friction forces as grains are pulled out in the crack wake (Vekinis et al., 1990). Residual stresses acting parallel to the crack plane have a strong influence on this process, since the friction forces are caused in part by local compressive stresses acting between a grain and the surrounding matrix. A more detailed model of this process has been given by Bower and Ortiz (1991).

\section{Summary and Conclusions}

The toughening enhancement due to residual stresses in a polycrystalline ceramic has been estimated, using a theoretical model. The residual stress was idealized as a doubly sinusoidal distribution, amplitude $\sigma_{r}$, and wavelength $L$, acting perpendicular to the plane of an initially straight semi-infinite crack. Only mode I stress intensities are induced by this stress, so the crack is not deflected from its initial plane. However, regions of compressive stress trap the crack front, causing it to bow out between them. The crack shape has been calculated for a range of residual stress amplitudes, using an incremental perturbation method developed by Bower and Ortiz (1990), suitably extended to account for nonuniform remote stresses. It has been shown that some transient toughening is produced by the residual stresses. Furthermore, if the stress amplitude exceeds $\sigma_{r} \sqrt{L} / K_{c}^{\text {mat }}=2.1$, pinning particles are left behind in the crack wake. These results confirm the predictions of Evans et al. (1977).

However, for values of residual stress and grain size representative of a typical ceramic, the predicted toughness en- hancement is less than one percent. Furthermore, no pinning particles are formed. It is concluded that the R-curve behavior and bridging particles observed in monolithic ceramics are not caused by residual stresses acting perpendicular to the crack plane.

\section{References}

Blendell, J. E., and Coble, R. L., 1982, "Measurement of Stress Die t Thermal Expansion Anisotropy in $\mathrm{Al}_{2} \mathrm{O}_{3}$," J. Am. Ceram. Soc., Vol. 65, No 3, pp. 174-183.

Bower, A. F., and Ortiz, M., 1990, "Solution of Three-Dimensional Cract Problems by a Finite Perturbation Method," Journal of the Mechanics and Physics of Solids, Vol. 38, No. 4, pp. 443-480.

Bower, A. F., and Ortiz, M., 1991, "A Three-Dimensional Analysis of Cract Trapping and Bridging by Tough Particles," Journal of the Mechanics and Physics of Solids, Vol. 39, No. 6, pp. 815-858.

Budiansky, B., 1986, "Micromechanics II," Proc. 10th National U.S. Con gress of Applied Mechanics, P. J. Lamb, ed., Austin, Texas, pp. 25-32.

Budiansky, B., and Amazigo, J. C., 1989, "Toughening by Aligned, Fric tionally Constrained Fibres," Journal of the Mechanics and Physics of Solids Vol. 37, No. 1, pp. 93-109.

Budiansky, B., Amazigo, J. C., and Evans, A. G., 1988, "Small Scale Cracl Bridging and the Fracture Toughness of Particulate Reinforced Ceramics, Journal of the Mechanics and Physics of Solids, Vol. 36, No. 2, pp. 167-187

Budiansky, B., Hutchinson, J., and Evans, A. G., 1986, "Matrix Fracture in Fibre Reinforced Ceramics," Journal of the Mechanics and Physics of Solids Vol. 34, pp. 167-189.

Evans, A. G., Heuer, A. H., and Porter, D. L., 1977, "The Fracture Tougt ness of Ceramics," Advances in Research on the Strength and Fracture of Materials, ICF4, D.M.R. Taplin, ed.

Fares, N., 1989, "Crack Fronts Trapped by Arrays of Obstacles: Numerical Solutions Based on Surface Integral Representation," ASME JoURNAL of Ap. PLIED MeChanics, Vol. 56, pp. 837-843.

Gao, H., and Rice, J. R., 1986, "Shear Stress Intensity Factors for a Planar Crack with Slightly Curved Front," ASME Journal of APPLIED MEchanics, Vol. 53, pp. 774-778.

Gao, H., and Rice, J. R., 1987, "Somewhat Circular Tensile Cracks," In ternational Journal of Fracture, Vol. 33, pp. 115-174.

Gao, H., and Rice, J. R., 1989, "A First-Order Perturbation Analysis of Crack Trapping by Arrays of Obstacles," ASME Journal of APpliED MeCHANICS, Vol. 56, pp. 828-836.

Krstic, V. V., Nicholson, P. S., and Hoagland, R. G., 1981, "Toughening of Glasses by Metallic Particles," Journal of the American Ceramics Society. Vol. 64, No. 9, pp. 499-503.

Mendelson, M. 1., and Fine, M. F., 1974, "Enhancement of Fracture Prop. erties of Wustite by Precipitation," Journal of the American Ceramics Societv. Vol. 57, No. 4, pp. 154-159.

Ortiz, M., and Molinari, A., 1988, "Microstructural Thermal Stresses in Ceramic Materials," Journal of the Mechanics and Physics of Solids, Vol 36, pp. 385-400.

Ortiz, M., and Suresh, S., 1993, "Residual Stresses in Ceramics due to Thermal and Elastic Anisotropy: Statistical Properties and Microfracture," ASME JotrNAL OF APPLIED MeChaniCs, in press.

Rice, J. R., 1985, "First Order Variation in Elastic Fields due to Variation in Location of a Planar Crack Front," ASME Journal of APPLIED MEchanics. Vol. 52, pp. 571-579.

Rice, J. R., 1989, "Weight Function Theory for Three-Dimensional Elastic Crack Analysis," Fracture Mechanics: Perspectives and Directions (Twentieth Symposium), ASTM-STP-1020, R. P. Wei and R. P. Gangloff, eds., American Society Testing and Materials, Philadelphia, pp. 29-57.

Sigl, L. S., and Evans, A. G., 1989, "Effects of Residual Stress and Frictional Sliding on Cracking and Pull Out in Brittle Matrix Composites," Mechanics of Materials, Vol. 8, pp. 1-12.

Swanson, P. L., Fairbanks, C. J., Lawn, B. R., Mai, Y. W., and Hockey. B. J., 1987, "Crack Interface Grain Bridging as a Fracture Resistance Mechanism in Ceramics I: Experimental Study on Alumina," Journal of the Americon Ceramics Society, Vol. 70, No. 4, pp. 279-289.

Vekinis, G., Ashby, M. F., and Beaumont, P. W. R., 1990, "R-Curve Behavior of $\mathrm{Al}_{2} \mathrm{O}_{3}$ Ceramics," Acta Metall., Vol. 38, No. 6, pp. 1151-1162. 